\title{
Comparison of abdominal muscle activity during abdominal drawing-in maneuver combined with irradiation variations
}

\author{
Young-In Hwang', Du-Jin Park ${ }^{2, *}$ \\ 'Department of Physical Therapy, College of Biohealth Science, Hoseo University, Asan, Korea \\ 2Department of Physical Therapy, Kaya University, Gimhae, Korea
}

\begin{abstract}
Many experts have used an indirect method for enhancing strength and performance of muscles in clinical practice. The indirect method, which called an irradiation is a basic procedure of proprioceptive neuromuscular facilitation, there is little research related the effects of irradiation. This study investigated abdominal muscle activity during abdominal drawing-in maneuver (ADIM) combined with irradiation variations. The study recruited 42 healthy, young adults who were divided randomly into three groups according to which intervention they received. The first group performed the ADIM combined with coactivation of the pelvic floor muscle. The second group performed the ADIM combined with the irradiation resulting from dorsiflexion of the ankle. The third group performed the ADIM combined with the irradiation resulting from bilateral arm extension. Electromyography data were collected from the
\end{abstract}

rectus abdominis, external oblique abdominis, and transversus abdominis/internal oblique abdominis ( $\mathrm{Tr} A / \mathrm{IO}$ ) muscles during ADIM combined with irradiation variations. There were significant differences in the abdominal muscle activity and the preferential contraction ratio of the TrA/ 10 among the three groups $(P<0.05)$. ADIM combined with irradiation resulting from bilateral arm extension may be effective for enhancing the deep and superficial abdominal muscles of healthy people and athletes. The ADIM without the irradiation is advantageous for recovering motor control of the TrA/IO.

Keywords: Proprioceptive neuromuscular facilitation, Irradiation, Abdominal drawing-in maneuver

\section{INTRODUCTION}

Proprioceptive neuromuscular facilitation (PNF) is a concept of training widely used in clinical practice in order to improve the performance of the neuromusculoskeletal system by stimulation of muscle and joint proprioceptors using basic procedures (Gontijo et al., 2012; Kofotolis and Kellis, 2007). PNF is recognized as a stretching technique for muscle elasticity and shown to have a positive effect on active and passive range of motion (Hindle et al., 2012; Sharman et al., 2006). Additionally, this often used as an alternative form of progressive resistive exercise by physiotherapist or athletic trainer as its use has a greater benefit than general strength training in athletic injury rehabilitation (Lusting et al., 1992).

The irradiation is a basic procedure of PNF, which is defined as increasing the spread and strength of the response to resistance (Shimura and Kasai, 2002), an outcome that might derive from resistance-induced temporal or spatial summation (Eccles and Sherrington, 1930). This is based on the fact that the stimulation of strong, healthy muscles increases the activity of the contralateral injured muscles and the number of active motor units recruited and weakens the neuromuscular response (Adler et al., 2008; Shimura and Kasai, 2002).

When it is difficult to train the injured areas directly in sport rehabilitation, the cross training that improves strength of injured part using the opposite part is a form of irradiation (Abreu et al., 2015; Kofotolis and Kellis, 2007; Reznik et al., 2015). The

*Corresponding author: Du-Jin Park (iD http://orcid.org/0000-0002-5658-3343 Department of Physical Therapy, Kaya University, 208 Samgye-ro, Gimhae 50830, Korea

Tel: +82-55-330-1047, Fax: +82-55-344-5285, E-mail: djpark35@kaya.ac.kr Received: May 29, 2017 / Accepted: June 10, 2017
This is an Open Access article distributed under the terms of the Creative Commons Attribution Non-Commercial License (http://creativecommons.org/licenses/by-nc/4.0/) which permits unrestricted non-commercial use, distribution, and reproduction in any medium, provided the original work is properly cited. 
mechanism is uncertain and may be caused by the bilateral distribution of the descending motor pathways, neural overflow and muscular system (Kofotolis and Kellis, 2007; Røe et al., 2000). The irradiation can be occurred through various body parts as well as the form of cross training. The application of irradiation is achieved in various ways, not only for cross training. First of all, it is used to improve the muscle strength of distal part through the proximal movement. There are some studies that irradiation resulting from trunk was effective to increase the muscle strength of lower extremity (Gontijo et al., 2012; Tayashiki et al., 2016).

On the contrary, it is able to improve the muscle strength of proximal through the distal movement. Previous study reported that the abdominal drawing-in maneuver (ADIM) combined with the irradiation resulting from ankle dorsiflexion has improved the function of transversus abdominis ( $\operatorname{Tr} A)$ (Chon et al., 2010). Although recent studies have examined the effects of irradiation using ankle motion, few studies have identified the effects of irradiation resulting from upper extremity on the strength of abdominal muscle. Therefore, this study investigated abdominal muscle activity during ADIM combined with irradiation variations.

\section{MATERIALS AND METHODS}

\section{Subjects}

This study recruited 42 health adults (27 male and 15 female adults). They were randomly divided into three groups of 14 according to the exercise intervention used. The study was approved by the Research Ethics Committee of Kaya University (Kaya IRB-146). Participants were excluded from the study if they had symptoms of musculoskeletal or neurological disease, functional limitations, pain or dysfunction in the upper or lower extremities on exercise, back pain during the last 6 months, or experience with the ADIM.

\section{Instrumentation}

We used surface electromyography (Free EMG 300, BTS Bioengineering, Garbagnate Milanese, Italy) to record and process the electromyography signals of the rectus abdominis (RA), external oblique abdominis (EO), and transversus abdominis/internal oblique abdominis ( $\mathrm{Tr} A / \mathrm{IO}$ ). The bandwidth of the surface electromyography was 20 to $500 \mathrm{~Hz}$; the sampling rate was set to 1,000 $\mathrm{Hz}$. Surface electrodes were attached to the muscle fibers on right sides of the body: for the RA, the electrodes were attached $1 \mathrm{~cm}$ laterally and $2 \mathrm{~cm}$ inferiorly from the navel; for the EO, they were attached diagonally to the lower edge of the line connecting the contralateral pubic symphysis and the lowest edge of the rib $(\mathrm{Ng}$ et al., 1998). Although Lima et al. (2012) recommended using an internal electrode to observe the activity of the $\operatorname{Tr} A$, the Research Ethics Committee recommended changing the method to avoid pain and infection in the subjects. Therefore, we observed the TrA/ IO using electrodes attached $2 \mathrm{~cm}$ medially and $2 \mathrm{~cm}$ inferiorly to the anterior superior iliac spine (Marshall and Murphy, 2003).

To standardize the action potential of each muscle, a maximal voluntary isometric contraction (MVIC) was performed against manual resistance. The subjects lay flat on their backs, with their knees bent to $90^{\circ}$, and both hands crossed on the chest. The $\operatorname{Tr} \mathrm{A} /$ IO was measured when the subjects turned their trunks to the left while curving their trunks. The EO was measured when the subjects turned their trunks to the opposite side while curving their trunks. The RA was measured when the subjects curved their trunks without turning them while crossing the arms. To prevent muscle fatigue while the MVIC was measured, the subjects maintained the contraction for $5 \mathrm{sec}$ and then rested for $30 \mathrm{sec}$. We measured each MVIC 3 times, and used the mean value for the middle 3 sec.

\section{Tasks and procedures}

For the ADIM, the participants were asked to draw in their abdomen without moving the spine, ribs, or pelvis, while gradually pulling their navel inward and toward their heads. During the ADIM, a pressure biofeedback unit (Stabilizer, Chattanooga Medical Supply Inc., Chattanooga, TN, USA) was located between the subject's waist and the ground surface, whereas the pressure gauge was maintained at $40-42 \mathrm{mmHg}$ (Park and Lee, 2013). To contract the pelvic floor muscle, the women were asked to perform a contraction as if holding urine by pulling the inner muscles of the vagina inward and tightening them; the men were asked to perform a contraction that felt like they were flexing the penis (Norris, 1999).

Group A performed the ADIM combined with coactivation of the pelvic floor muscle (Fig. 1A). Group B performed the ADIM combined with the irradiation resulting from dorsiflexion of the ankle (Fig. 1B). Group $C$ performed the ADIM with the irradiation resulting from bilateral arm extension (Fig. 1C). We used a muscle testing device (Commander Muscle Testing, J-tech Inc., Chester Springs, PA, USA) to maintain constant resistance-provoking irradiation. For resistance to the irradiation, the maximum contraction was measured by the device, and resistance equal to approximately $70 \%$ of the maximum contraction was applied.

The participants were asked to maintain the abdominal con- 
traction for $10 \mathrm{sec}$ while breathing normally. Each posture was performed three times. To prevent muscle fatigue caused by continuous measurements, the exercise intervention was performed for $10 \mathrm{sec}$ followed by a 1-min rest. The muscle activity based on the exercise intervention was calculated as the mean value for 6-sec excluding the first and last 2 sec.
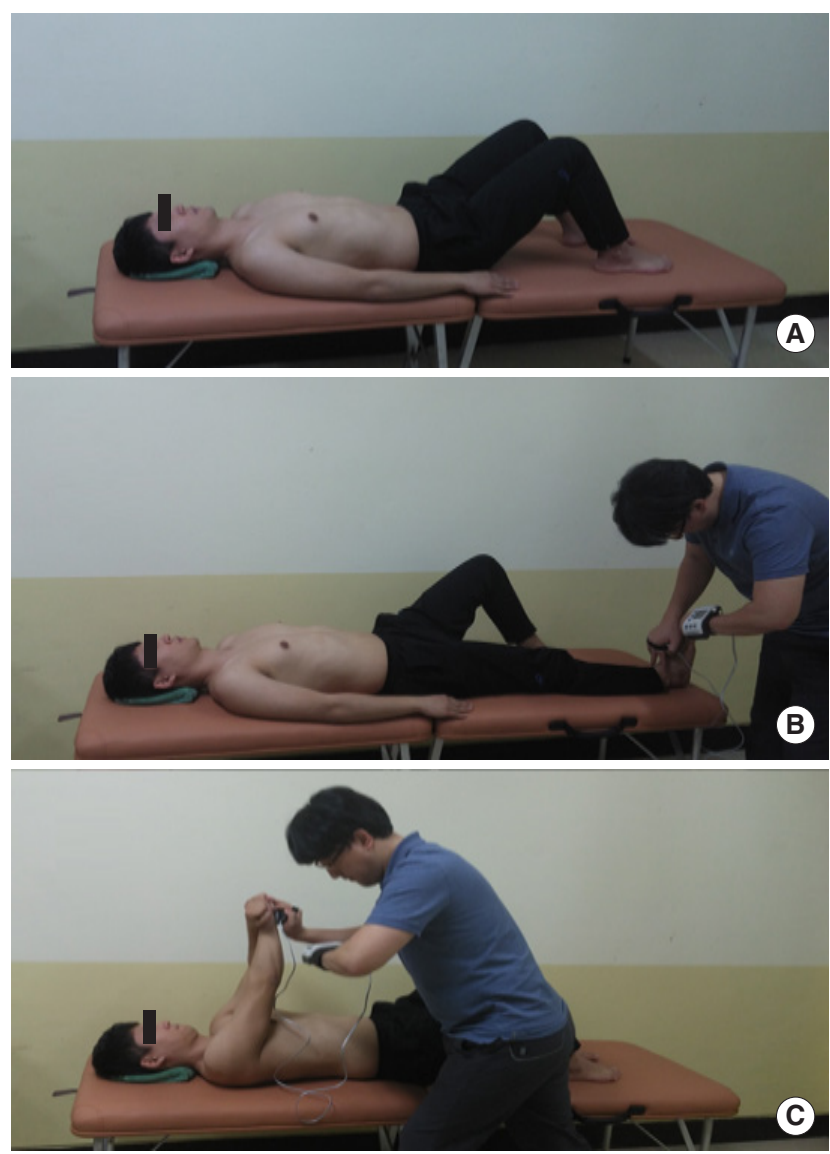

Fig. 1. Abdominal drawing-in maneuver (ADIM): (A) ADIM combined with coactivation of pelvic floor muscle, (B) ADIM combined with the irradiation resulting from dorsiflexion of the ankle, (C) ADIM combined with the irradiation resulting from bilateral arm extension.

\section{Statistical analysis}

Forty-two participants were required for the present study design, which set as $80 \%$ of power, 0.5 of effect size $\left(\eta^{2}\right)$, and 0.05 of alpha level. One-way analysis of variance was used to compare the differences in the actions of the abdominal muscles and the preferential contraction ratio (PCR) of the $\operatorname{Tr} \mathrm{A} / \mathrm{IO}$ according to the three interventions. Scheffé multiple comparison analysis was used as a posttest. IBM SPSS ver. 18.0 (IBM Co., Armonk, NY, USA) was used for the statistical analyses. The significance level was set at $\alpha=0.05$.

\section{RESULTS}

The mean age, height, body weight, and body mass index of the subjects were $21.36 \pm 1.64$ years, $169.9 \pm 8.52 \mathrm{~cm}, 64.33 \pm 13.50$ $\mathrm{kg}$, and $22.16 \pm 3.76 \mathrm{~kg} / \mathrm{m}^{2}$, respectively. These values do not represent significant differences among the three groups.

Table 1 shows the activities of the abdominal muscles associated with the three interventions. Significant differences among the three groups were found in the RA, EO, and $\operatorname{Tr} \mathrm{A} / \mathrm{IO}$. The abdominal muscles were significantly more active in members of group C (Fig. 2). The PCR of the $\operatorname{TrA} / \mathrm{IO}$ differed significantly among the three groups and was highest in group A (Fig. 2).

\section{DISCUSSION}

It has been reported that the ADIM combined with the irradiation using dorsiflexion of the ankle resulted in significantly greater activity of the $\operatorname{Tr} A$ when compared with the standard ADIM (Chon et al., 2010); however, no significant difference in this regard was observed in the current study. These results may relate to differences between the standard ADIM and the ADIM accompanied by a contraction of the pelvic floor muscle. Contraction of the pelvic floor muscle interacts with the $\operatorname{Tr} A$ to increase the activity

Table 1. Changes in the abdominal muscle activity and the TrA/IO PCR during abdominal drawing-in maneuver combined with irradiation variations

\begin{tabular}{lrrrr}
\hline Variable & Group A & Group B & Group C & $F$ \\
\hline RA $^{*}(\% M V I C)$ & $5.58 \pm 1.69$ & $7.51 \pm 1.00$ & $13.26 \pm 3.00$ & 52.111 \\
EO $(\% M V I C)$ & $10.91 \pm 4.36$ & $14.07 \pm 3.43$ & $20.88 \pm 2.96$ & 27.620 \\
TrA/IO* $(\% M V I C)$ & $21.69 \pm 3.52$ & $24.22 \pm 3.61$ & $41.12 \pm 8.92$ & 44.621 \\
TrA/IO PCR* & $0.57 \pm 0.05$ & $0.53 \pm 0.03$ & $0.54 \pm 0.03$ & 4.852 \\
\hline
\end{tabular}

Values are presented as mean \pm standard deviation.

Group A, abdominal drawing-in maneuver (ADIM) combined with coactivation of pelvic floor muscle; group B, ADIM combined with the irradiation resulting from dorsiflexion of the ankle; group C, ADIM combined with the irradiation resulting from bilateral arm extension; TrA/IO, transversus abdominis/internal oblique abdominis; PCR, preferential contraction ratio; RA, rectus abdominis; EO, extenal oblique abdominis; MVIC, maximal voluntary isometric contraction.

*Significant difference between conditions. 

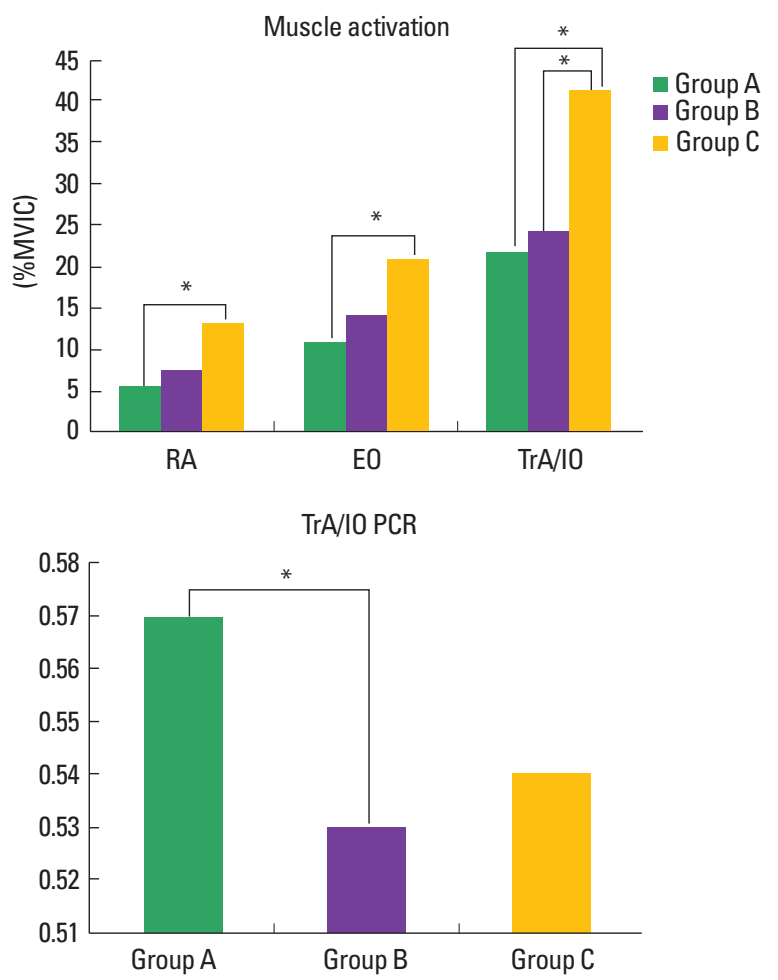

Fig. 2. Changes in abdominal muscle activity and the TrA/IO PCR among three groups. Group A, ADIM combined with co-activation of pelvic floor muscle; group $B, A D I M$ combined with the irradiation resulting from dorsiflexion of the ankle; group $\mathrm{C}, \mathrm{ADIM}$ combined with the irradiation resulting from bilateral arm extension; RA, rectus abdominis; E0, extenal oblique abdominis; $\operatorname{TrA} / \mathrm{IO}$, transversus abdominis/internal oblique abdominis; PCR, preferential contraction ratio; MVIC, maximal voluntary isometric contraction. *Significant difference between conditions.

of the latter and to provide trunk stability by enhancing the abdominal pressure by about $6 \mathrm{mmHg}$ (Neumann and Gill, 2002; Sapsford and Hodges, 2001). Some studies have reported that the ADIM combined with pelvic floor muscle contraction was more effective than the traditional ADIM at increasing the TrA activity (Critchley, 2002; Mew, 2009).

Based on the results of the preceding studies, the ADIM combined with coactivation of the pelvic floor muscle increased the TrA activity compared with the traditional ADIM. Additionally, the muscle activities were similar to those of the ADIM combined with the irradiation resulting from dorsiflexion of the ankle. This suggests that the ADIM combined with coactivation of the pelvic floor muscle can increase the $\operatorname{Tr} \mathrm{A}$ actions to the same extent as the ADIM combined with the irradiation resulting from dorsiflexion of the ankle.

The ADIM combined with the irradiation resulting from bilateral arm extension induced significant increases in the activities of the $\operatorname{Tr} \mathrm{A} / \mathrm{IO}$ and the other abdominal muscles compared with the other two interventions. These results support the literature (Adler et al., 2008) that training using bilateral extremities was effective to facilitate the trunk muscles. The extension-adduction-external rotation patterns of the PNF applied to the upper extremities can activate the serratus, rhomboid, and pectoralis minor muscles (Adler et al., 2008) while stimulating the spiral lines of the fascia that connect the rhomboids, serratus muscle, and IO/EO to the pelvis (Myers, 2012). Then, the TrA can be activated indirectly because the lower fibers of the $\mathrm{IO}$ work together without separation of the TrA and fascia (Marshall and Murphy, 2003). Additionally, the upper extremity pattern of the PNF applied to both sides may generate movement for the curving of the trunk, summing the actions of the abdominal muscles.

The original purpose of the ADIM was to promote the preferential contraction of the deep $\operatorname{Tr} A$ while minimizing the contraction of the abdominal muscles in the surface layer. However, many studies have reported that it is difficult to remove the actions of the EO during the ADIM (Chanthapetch et al., 2009; Urquhart et al., 2005), and all types of intervention used in the current study produced similar results. The ADIM combined with coactivation of the pelvic floor muscles resulted in a higher PCR of the $\mathrm{Tr} A / \mathrm{IO}$ than did the ADIM combined with either of the irradiations. These results suggest that the irradiation are useful for increasing the activities of all the abdominal muscles but have a limited effect on the actions of the $\operatorname{Tr} A$ only.

The ADIM combined with the irradiation using bilateral arm extension is recommended for athletes or healthy, normal people rather than patients with early back pain, because it can enhance general muscle strength and reinforce contraction of the TrA. The ADIM combined with contraction of the pelvic floor muscles may be appropriate for improving the motor control ability and contraction ratio of the $\operatorname{Tr} A$ rather than its strength.

This study had several limitations. First, because the participants were 42 healthy adults, the generalizability of the results to adults of all ages is limited. Second, noninvasive surface electromyography has a limited ability to measuring the $\operatorname{Tr} A$ and the IO separately. Third, it is difficult to predict long-term changes based on this short-term study.

\section{CONFLICT OF INTEREST}

No potential conflict of interest relevant to this article was reported. 


\section{ACKNOWLEDGMENTS}

This work was supported by Kaya University Research Fund.

\section{REFERENCES}

Abreu R, Lopes AA, Sousa AS, Pereira S, Castro MP. Force irradiation effects during upper limb diagonal exercises on contralateral muscle activation. J Electromyogr Kinesiol 2015;25:292-297.

Adler SS, Beckers D, Buck M. PNF in practice: an illustrated guide. 3rd ed. Heidelberg (Germany); Springer Medizin Verlag; 2008.

Chanthapetch P, Kanlayanaphotporn R, Gaogasigam C, Chiradejnant A. Abdominal muscle activity during abdominal hollowing in four starting positions. Man Ther 2009;14:642-646.

Chon SC, Chang KY, You JS. Effect of the abdominal draw-in manoeuvre in combination with ankle dorsiflexion in strengthening the transverse abdominal muscle in healthy young adults: a preliminary, randomised, controlled study. Physiotherapy 2010;96:130-136.

Critchley D. Instructing pelvic floor contraction facilitates transversus abdominis thickness increase during low-abdominal hollowing. Physiother Res Int 2002;7:65-75.

Eccles JC, Sherrington CS. Reflex summation in the ipsilateral spinal flexion reflex. J Physiol 1930;69:1-28.

Gontijo LB, Pereira PD, Neves CD, Santos AP, Machado Dde C, Bastos $\mathrm{VH}$. Evaluation of strength and irradiated movement pattern resulting from trunk motions of the proprioceptive neuromuscular facilitation. Rehabil Res Pract 2012;2012:281937.

Hindle KB, Whitcomb TJ, Briggs WO, Hong J. Proprioceptive Neuromuscular Facilitation (PNF): Its Mechanisms and Effects on Range of Motion and Muscular Function. J Hum Kinet 2012;31:105-113.

Kofotolis ND, Kellis E. Cross-training effects of a proprioceptive neuromuscular facilitation exercise programme on knee musculature. Phys Ther Sport 2007;8:109-116.

Lima PO, Oliveira RR, Moura Filho AG, Raposo MC, Costa LO, Laurentino GE. Concurrent validity of the pressure biofeedback unit and surface electromyography in measuring transversus abdominis muscle activity in patients with chronic nonspecific low back pain. Rev Bras Fisioter 2012;16:389-395.

Lusting SA, Ball TE, Looney M. A comparison of two proprioceptive neuromuscular facilitation techniques for improving range of motion and muscular strength. Isokinet Exerc Sci 1992;2:154-159.
Marshall P, Murphy B. The validity and reliability of surface EMG to assess the neuromuscular response of the abdominal muscles to rapid limb movement. J Electromyogr Kinesiol 2003;13:477-489.

Mew R. Comparison of changes in abdominal muscle thickness between standing and crook lying during active abdominal hollowing using ultrasound imaging. Man Ther 2009;14:690-695.

Myers TW. Anatomy trains: myofascial meridians for manual and movement therapists. Edinburgh: Churchill Livingstone Elsevier; 2012.

Neumann P, Gill V. Pelvic floor and abdominal muscle interaction: EMG activity and intra-abdominal pressure. Int Urogynecol J Pelvic Floor Dysfunct 2002;13:125-132.

$\mathrm{Ng} \mathrm{JK}$, Kippers V, Richardson CA. Muscle fibre orientation of abdominal muscles and suggested surface EMG electrode positions. Electromyogr Clin Neurophysiol 1998;38:51-58.

Norris C. Functional load abdominal training: part 2. J Bodyw Mov Ther 1999;3:208-214

Park DJ, Lee SK. What is a suitable pressure for the abdominal drawing-in maneuver in the supine position using a pressure biofeedback unit? J Phys Ther Sci 2013;25:527-530.

Reznik JE, Biros E, Bartur G. An electromyographic investigation of the pattern of overflow facilitated by manual resistive proprioceptive neuromuscular facilitation in young healthy individuals: a preliminary study. Physiother Theory Pract 2015;31:582-586.

Røe C, Brox JI, Saugen E, Vøllestad NK. Muscle activation in the contralateral passive shoulder during isometric shoulder abduction in patients with unilateral shoulder pain. J Electromyogr Kinesiol 2000;10: 69-77.

Sapsford RR, Hodges PW. Contraction of the pelvic floor muscles during abdominal maneuvers. Arch Phys Med Rehabil 2001;82:1081-1088.

Sharman MJ, Cresswell AG, Riek S. Proprioceptive neuromuscular facilitation stretching: mechanisms and clinical implications. Sports Med 2006;36:929-939.

Shimura K, Kasai T. Effects of proprioceptive neuromuscular facilitation on the initiation of voluntary movement and motor evoked potentials in upper limb muscles. Hum Mov Sci 2002;21:101-113.

Tayashiki K, Maeo S, Usui S, Miyamoto N, Kanehisa H. Effect of abdominal bracing training on strength and power of trunk and lower limb muscles. Eur J Appl Physiol 2016;116:1703-1713.

Urquhart DM, Hodges PW, Allen TJ, Story IH. Abdominal muscle recruitment during a range of voluntary exercises. Man Ther 2005;10: 144-153. 